\title{
Nonlinear stability of the one domain approach to modelling convection in superposed fluid and porous layers
}

\author{
BY ANTONY A. HILL ${ }^{1}$ AND MAGDA CARR ${ }^{2}$ \\ ${ }^{1}$ School of Mathematical Sciences, University of Nottingham, Nottingham, \\ $N G 7$ 2RD, UK \\ ${ }^{2}$ School of Mathematics and Statistics, University of St Andrews, St Andrews, \\ KY16 9SS, UK
}

Studies of the nonlinear stability of fluid/porous systems have been developed very recently. A two domain modelling approach has been adopted in previous works but was restricted to specific configurations. The extension to the more general case, of a Navier-Stokes modelled fluid over a porous material, was not achieved for the two domain approach due to the difficulties associated with handling the interfacial boundary conditions. This paper addresses this issue by adopting a one domain approach, where the governing equations for both regions are combined into a unique set of equations which are valid for the entire domain. It is shown that the nonlinear stability bound, in the one domain approach, is very sharp and hence excludes the possibility of subcritical instabilities. Moreover, the one domain approach is compared to an equivalent two domain approach and excellent agreement is found between the two.

Keywords: superposed porous-fluid convection; one domain approach; energy method

\section{Introduction}

The stability of thermal convection in a system composed of a fluid overlying a porous medium saturated with the same fluid remains a subject of particular attention (cf. Nield \& Bejan 2006), due to the wide range of geophysical and industrial applications.

The main approach adopted in the literature to study this configuration is that of a two domain model, where the set of governing equations for each region are considered separately, with appropriate boundary conditions at the interface, cf. Ochoa-Tapia \& Whitaker (1995), Alazmi \& Vafai (2001), Goyeau et al. (2003), Chandesris \& Jamet (2006, 2009), Nield \& Bejan (2006), Hirata et al. (2007a). These models generally use Darcy's law (or Darcy-Brinkman for high porosities, cf. Nield \& Bejan 2006) in the porous region, coupled with the Navier-Stokes equations in the fluid region. Recent contributions include Vafai (2005), Chang (2006), Mu \& Xu (2007), Straughan (2008), Hill \& Straughan (2009) and Hill \& Carr (2010).

An alternative, less widely used approach, is that of a one domain model, where the governing equations for both regions are combined into a unique set of equations, which are valid for the entire domain. The physical properties which change the 
governing equations for each region are then taken to be discontinuous functions, see e.g. Hirata et al. (2009a). Comparisons between the one and two domain approaches, and the subsequent treatment of the interfacial region is discussed in Goyeau et al. (2003) and Hirata et al. (2007b). The equivalence of the one and two domain approaches for the Darcy-Brinkman/Navier Stokes model is shown in Hirata et al. (2009b).

Although linear instability has been studied extensively for fluid/porous systems, the exploration of nonlinear stability has been a much more recent development. Payne \& Straughan (1998) and Hill \& Straughan (2009) developed the first nonlinear stability thresholds for a fluid-porous system. In both these papers, owing to the difficulties associated with incorporating the nonlinear $\mathbf{v} \cdot \nabla \mathbf{v}$ advection term in the Navier-Stokes equations into a stability analysis, the fluid was modeled using Stokes equations. Hill \& Carr (2010) utilized a viscoelastic model proposed by Ladyzhenskaya (Ladyzhenskaya 1969; Straughan 2008) as an alternative to NavierStokes, and constructed nonlinear stability thresholds.

These previous papers on nonlinear stability adopt a two domain approach, which restricts their models to specific systems (i.e. Stokes flow and a viscoelastic fluid) due to the difficulty in incorporating the boundary conditions in a stability analysis. A one domain approach overcomes this difficulty by avoiding the explicit formulation of the boundary conditions at the fluid/porous interface. However, as the physical parameters which change between the regions are modelled using discontinuous functions, this precludes the use of a nonlinear generalised energy stability analysis, as one can not utilise the divergence theorem, cf. Straughan (2004).

In this paper we approximate these discontinuous functions with exponentially converging continuous ones, essentially making the relevant physical parameters homogeneous throughout the layers, with a small heterogeneous transition zone at the interface. By adopting this approach we show that it is possible to construct unconditional nonlinear energy stability thresholds, and compare this result with the linear instability of both the one, and two domain approaches. A comparison of the linear/nonlinear thresholds allows for the assessment of the suitability of linear theory to predict the physics of the onset of convection, which is crucial in understanding and controlling the system under investigation. This is discussed in more detail in $\S 3$. 


\section{Formation of the problem}

Consider a Newtonian fluid occupying the three-dimensional layer $\left\{(x, y) \in \mathbb{R}^{2}\right\} \times$ $\{z \in(0, d)\}$, which saturates an underlying homogeneous porous layer $\{(x, y) \in$ $\left.\mathbb{R}^{2}\right\} \times\left\{z \in\left(-d_{m}, 0\right)\right\}$. The interface between the saturated porous medium and the fluid layer is at $z=0$, see Figure 1 .

$$
z=d \quad T_{U}
$$

Fluid region

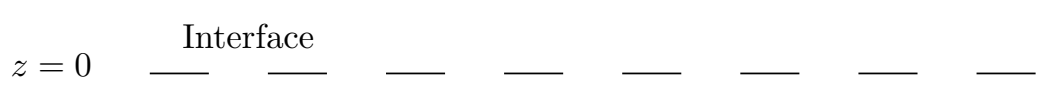

Porous medium

$z=-d_{m}$

Figure 1. Schematic diagram of a fluid region overlying a porous medium with the interface at $z=0$, where the upper and lower planes are impermeable and are kept at temperatures $T_{U}$ and $T_{L}$, respectively, with $T_{U}<T_{L}$.

The governing equations for both regions may be written in the form

$$
\begin{aligned}
& \frac{\rho_{0}}{\phi}\left(\frac{\partial v_{i}}{\partial t}+v_{j} \frac{\partial}{\partial x_{j}}\left(\frac{v_{i}}{\phi}\right)\right)=-\frac{\partial p}{\partial x_{i}}-\frac{\mu}{K} v_{i}+\frac{\partial}{\partial x_{j}}\left(\frac{\mu}{\phi}\left(\frac{\partial v_{i}}{\partial x_{j}}+\frac{\partial v_{j}}{\partial x_{i}}\right)\right) \\
& \quad-g \rho_{0} k_{i}\left(1-\alpha\left(T-T_{0}\right)\right), \\
& \frac{\partial v_{i}}{\partial x_{i}}=0 \\
& G \frac{\partial T}{\partial t}+v_{j} \frac{\partial T}{\partial x_{j}}=\frac{\partial}{\partial x_{j}}\left(\frac{\kappa}{\left(\rho_{0} c_{p}\right)_{f}} \frac{\partial T}{\partial x_{j}}\right)
\end{aligned}
$$

where the variables $v_{i}, p, T, c_{p}, \rho_{0}$ and $T_{0}$ are the velocity, pressure, temperature, specific heat at a constant pressure and reference density and temperature values, respectively. The porosity $\phi$, permeability $K$ and thermal diffusivity $\kappa$ take different values in the fluid and porous regions, see Table 1 , where $m$ and $f$ refer to the value of the parameter in the porous and fluid region, respectively. The quantities $\kappa_{m}$ and $G_{m}=\left(\rho_{0} c_{p}\right)_{m} /\left(\rho_{0} c_{p}\right)_{f}$ are defined in terms of the fluid and solid components of the porous medium, such that $Q_{m}=\phi Q_{f}+(1-\phi) Q_{s}$, where $Q_{m}=\kappa_{m}$ or $\left(\rho_{0} c_{p}\right)_{m}$.

In the two domain approach, the set of governing equations for each region are considered separately and boundary conditions at the interface are used to couple the system. System (2.1) is equivalent to adopting the Navier-Stokes and the DarcyBrinkman equations to govern the flow in the fluid domain and the porous region, respectively, cf. Hirata et al. (2007b, 2009b).

In this paper we adopt the one domain approach, where the porous layer is viewed as a pseudo fluid, making the whole layer a continuum. System (2.1) then 
Table 1. Fluid/porous layer parameters

\begin{tabular}{c|c|c} 
Parameter & Porous Region & Fluid Region \\
\hline$\phi$ & $\phi_{m}$ & 1 \\
$K^{-1}$ & $K^{-1}$ & 0 \\
$G$ & $G_{m}$ & 1 \\
$\kappa$ & $\kappa_{m}$ & $\kappa_{f}$
\end{tabular}

forms a unique set of equations, which are valid for the entire domain, avoiding the explicit formulation of boundary conditions at the interface. The physical properties which vary between the two regions are described by Table 1 . A comprehensive discussion of the variances and various physical attributes regarding the governing equations is given in Alazmi \& Vafai (2000).

Letting discontinuous functions describe the changing parameters in Table 1, linear instability thresholds can be derived, cf. Goyeau et al. (2003), Hirata et al. (2007b, 2009b). However, this approach precludes the direct construction of a nonlinear stability threshold, as it prevents the use of the divergence theorem (which will be discussed in Section $3 \mathrm{~b}$ ). To address this issue, let us consider functions of the form

$$
F\left(F_{1}, F_{2}\right)=\frac{F_{2}+F_{1} \mathrm{e}^{-\frac{2 n z}{d m}}}{1+\mathrm{e}^{-\frac{2 n z}{d m}}} .
$$

Clearly as $n \rightarrow \infty$ the function becomes

$$
F=\left\{\begin{array}{ll}
F_{1} & -d_{m}<z<0 \\
\frac{F_{1}+F_{2}}{2} & z=0 \\
F_{2} & 0<z<d
\end{array} .\right.
$$

Under this definition we approximate the discrete variables by

$$
\phi=F\left(\phi_{m}, 1\right), \quad \frac{1}{K}=F\left(\frac{1}{K}, 0\right), \quad G=F\left(G_{m}, 1\right), \quad \kappa=F\left(\kappa_{m}, \kappa_{f}\right) .
$$

Due to the exponential nature of $F\left(F_{1}, F_{2}\right)$, these parmaters are essentially homogeneous throughout the layers (for large $n$, which we can choose at our discretion), with a small heterogeneous transition zone at the interface, see Figure 2.

Fixing the temperatures at the upper and lower boundaries to be $T_{U}$ and $T_{L}$, respectively, and assuming no slip at these surfaces, such that $v_{i}=0$ at $z=-d_{m}, d$, governing equations (2.1) admit a steady state solution in which the velocity field is zero and

$$
\frac{d \bar{T}}{d z}=-\frac{\left(T_{L}-T_{U}\right) \epsilon_{T} c}{d_{m}}\left(\frac{1+\mathrm{e}^{-\frac{2 n z}{d_{m}}}}{\epsilon_{T}+\mathrm{e}^{-\frac{2 n z}{d_{m}}}}\right)
$$

In these equations $\epsilon_{T}=\kappa_{f} / \kappa_{m}$ and $\hat{d}=d / d_{m}$, with the overbar denoting the steady state and

$$
c^{-1}=\hat{d}+1+\frac{1-\epsilon_{T}}{2 n} \log \left(\frac{\epsilon_{T}+\mathrm{e}^{-2 n \hat{d}}}{\epsilon_{T}+\mathrm{e}^{2 n}}\right) .
$$




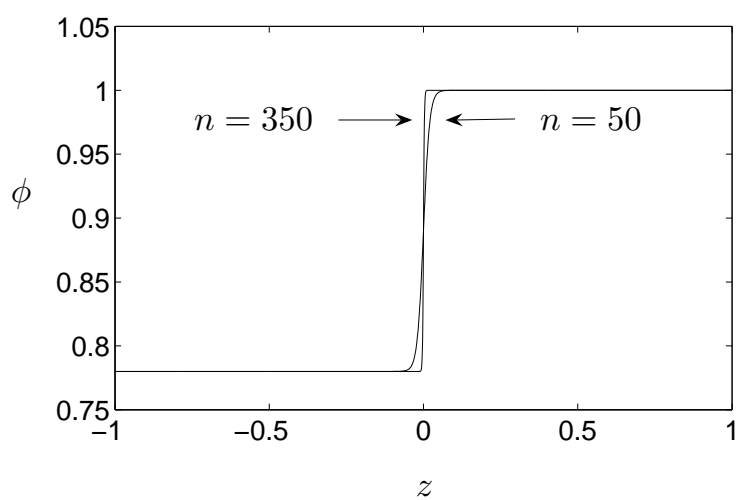

Figure 2. Visual representation of the function $\phi(z)=F\left(\phi_{m}, 1\right)$ for $n=50$ and $n=350$, with $\phi_{m}=0.78$ and $d=d_{m}=1$.

To assess the instability of the steady state we introduce perturbations to the steady solution of the form

$$
v_{i}=\bar{v}_{i}+u_{i}, \quad p=\bar{p}+\pi, \quad T=\bar{T}+\theta
$$

and non-dimensionalise with scalings of

$$
\begin{aligned}
& u_{i}=\frac{\mu}{\rho d_{m}} u_{i}^{*}, \quad \pi=\frac{\mu^{2}}{\rho_{0} d_{m}^{2}} \pi^{*}, \quad \theta=\theta^{*} \sqrt{\frac{\mu^{3}\left(T_{L}-T_{U}\right)\left(\rho_{0} c_{p}\right)_{f}}{\rho^{3} g \alpha d_{m}^{3} \kappa_{f}}}, \quad x_{i}=d_{m} x_{i}^{*}, \\
& t=\frac{\rho_{0} d^{2}}{\mu_{0}} t^{*}, \quad R=\sqrt{\frac{g \alpha \rho_{0} d_{m}^{3}\left(T_{L}-T_{U}\right)\left(\rho_{0} c_{p}\right)_{f}}{\mu \kappa_{f}}}
\end{aligned}
$$

where $R_{a}=R^{2}$ is the Rayleigh number. Substituting the perturbations and nondimensionalised variables into (2.1) (and dropping the stars), we derive the nondimensional perturbation equations

$$
\begin{aligned}
& \frac{1}{\phi}\left(\frac{\partial u_{i}}{\partial t}+u_{j} \frac{\partial}{\partial x_{j}}\left(\frac{u_{i}}{\phi}\right)\right)=-\frac{\partial \pi}{\partial x_{i}}-f_{1} u_{i}+\frac{\partial}{\partial x_{j}}\left(\frac{1}{\phi}\left(\frac{\partial u_{i}}{\partial x_{j}}+\frac{\partial u_{j}}{\partial x_{i}}\right)\right)+k_{i} R \theta \\
& \frac{\partial u_{i}}{\partial x_{i}}=0, \\
& \operatorname{Pr}\left(G \frac{\partial \theta}{\partial t}+u_{j} \frac{\partial \theta}{\partial x_{j}}\right)-R f_{2} c w=\frac{\partial}{\partial x_{j}}\left(\frac{1}{f_{2}} \frac{\partial \theta}{\partial x_{j}}\right),
\end{aligned}
$$

where $w=u_{3}, \operatorname{Pr}=\mu\left(\rho_{0} c_{p}\right)_{f} /\left(\kappa_{f} \rho_{0}\right), \delta=K / d_{m}^{2}$ is the Darcy number, $f_{1}=$ $F(1 / \delta, 0), f_{2}=1 / F\left(\varepsilon_{T}^{-1}, 1\right)$ and $F\left(F_{1}, F_{2}\right)=\left(F_{2}+F_{1} \mathrm{e}^{-2 n z}\right) /\left(1+\mathrm{e}^{-2 n z}\right)$.

\section{Stability Analysis}

The main impetus behind the use of a linear instability analysis on the thermal convection problem is to predict the onset of convection. To form such an analysis 
it is assumed that the perturbation is small and so neglects terms of quadratic and higher order, which leads to this approach providing limited information on the behavior of the nonlinear system. There is, therefore, the potential for regions of subcritical instabilities where the instability occurs prior to the thresholds predicted by the linear theory being reached.

To address this issue one can establish stability results through the use of generalised nonlinear energy techniques (cf. Straughan 2004). By comparing the instability thresholds generated by linear theory with the stability thresholds generated by the energy method, an assessment of the suitability of linear theory to predict the onset of convection can then be made. The energy method has been shown to be highly successful in thermal convection problems, where the symmetric part of linear operator associated with the governing equations dominates (cf. Straughan 2004).

\section{(a) Linear Instability Analysis}

The linearised equations are derived from $(2.2)_{1}$ and $(2.2)_{3}$ by discarding the nonlinear terms and assuming normal modes of the form

$$
u_{i}=u_{i}(z) \mathrm{e}^{\sigma t+i\left(a_{1} x+a_{2} y\right)}, \quad \pi=\pi(z) \mathrm{e}^{\sigma t+i\left(a_{1} x+a_{2} y\right)}, \quad \theta=\theta(z) \mathrm{e}^{\sigma t+i\left(a_{1} x+a_{2} y\right)},
$$

where $a_{1}, a_{2} \in \mathbb{R}$ are the horizontal wavenumbers and $\sigma \in \mathbb{C}$. Letting $D=d / d z$ and taking the double curl of $(2.2)_{1}$ to remove the pressure term, where the third component is chosen, leads to the linearised equations

$$
\begin{gathered}
\frac{1}{\phi}\left(D^{2}-a^{2}\right)^{2} w+D^{2}\left(\frac{1}{\phi}\right)\left(D^{2}+a^{2}\right) w+2 D\left(\frac{1}{\phi}\right) D\left(D^{2}-a^{2}\right) w- \\
D f_{1} D w-f_{1}\left(D^{2}-a^{2}\right) w-a^{2} R \theta=\sigma D\left(\frac{1}{\phi}\right) D w+\frac{\sigma}{\phi}\left(D^{2}-a^{2}\right) w \\
\frac{1}{f_{2}}\left(D^{2}-a^{2}\right) \theta+D\left(\frac{1}{f_{2}}\right) D \theta+R c f_{2} w=\sigma \operatorname{Pr} G \theta
\end{gathered}
$$

where $a^{2}=a_{1}^{2}+a_{2}^{2}$. The boundary conditions for the sixth order system at $z=-1$ and $\hat{d}$ are

$$
w=D w=\theta=0
$$

Numerical results for the linear instability of this one domain approach are presented in $\S 4$. Results for the equivalent two domain approach to linear instability (which are taken from Hill \& Straughan 2009) are also presented in $\S 4$ for comparison. As the continuous parameter approach is, essentially, an approximation to the discontinous model, a comparison with the standard approach is crucial to evaluate if our method captures the correct stability behaviour.

\section{(b) Nonlinear Stability Analysis}

To obtain global nonlinear stability bounds in the stability measure $L^{2}(\Omega)$, where $\Omega$ is the period cell for the perturbations, we multiply equations $(2.2)_{1}$ and 
$(2.2)_{3}$ by $u_{i}$ and $\theta$ respectively, and integrate over $\Omega$ to obtain

$$
\begin{aligned}
\int_{\Omega} \frac{1}{2} \frac{d}{d t} \frac{|\mathbf{u}|^{2}}{\phi}+ & \frac{u_{i} u_{j}}{\phi}\left(\frac{u_{i}}{\phi}\right)_{, j}+\pi_{, i} u_{i} d \Omega \\
=-\int_{\Omega} f_{1}|\mathbf{u}|^{2} & +\frac{\partial}{\partial x_{j}}\left(\frac{1}{\phi}\left(u_{i, j}+u_{j, i}\right)\right) u_{i}+R \theta w d \Omega \\
\operatorname{Pr} \int_{\Omega} \frac{G}{2} \frac{d}{d t}|\theta|^{2}+u_{j} \theta, j \theta d \Omega & =R c \int_{\Omega} f_{2} w \theta+\frac{\partial}{\partial x_{j}}\left(\frac{\theta, j}{f_{2}}\right) \theta d \Omega .
\end{aligned}
$$

Due to the exponential formulation taken in $\S 2, \phi, f_{1}, f_{2}$ and $G$ are continuous functions, allowing for the manipulation of the terms containing these functions using the divergence theorem, cf. Straughan (2004).

Letting $\lambda$ be a positive coupling parameter to be selected at our discretion, and defining an energy

$$
E(t)=\frac{1}{2} \int_{\Omega} \frac{|\mathbf{u}|^{2}}{\phi}+\lambda \operatorname{Pr} G|\theta|^{2} d \Omega
$$

we have the following identity:

$$
\frac{d E}{d t}=\mathcal{I}-\mathcal{D}
$$

where

$$
\begin{aligned}
\mathcal{I} & =\int_{\Omega}\left(1+c \lambda f_{2}\right) w \theta d \Omega \\
\mathcal{D} & =\int_{\Omega} f_{1}|\mathbf{u}|^{2}+\frac{1}{\phi}\left(u_{i, j}+u_{j, i}\right) u_{i, j}+\frac{\lambda}{f_{2}}|\nabla \theta|^{2} d \Omega .
\end{aligned}
$$

Utilising Poincaré like inequalities (cf. Payne \& Straughan 1998, Hill \& Carr 2010) it follows that $\mathcal{D} \geq c E$ for some positive constant $c$. Letting $1 / R_{E}=\max _{\mathcal{H}}(\mathcal{I} / \mathcal{D})$, where $\mathcal{H}$ is the space of admissible functions, yields

$$
\frac{d E}{d t} \leq c\left(\frac{R_{E}-R}{R_{E}}\right)
$$

if $R_{E}>R$. Integrating, we have $E(t) \leq \exp \left(-c\left(R_{E}-R\right) t / R\right)$, which tends to 0 as $t \rightarrow \infty$, if $R_{E}-R>0$, so that convergence is at least exponential. Therefore unconditional nonlinear stability can be established for $R_{E}>R$.

At the sharpest threshold $R_{E}=R$, the maximisation problem $\max _{\mathcal{H}}(\mathcal{I} / \mathcal{D})$ is solved by the Euler-Lagrange equations

$$
\begin{aligned}
R k_{i}\left(1+c \lambda f_{2}\right) \theta-2 f_{1} u_{i}+2 \frac{\partial}{\partial x_{j}}\left(\frac{1}{\phi}\left(u_{i, j}+u_{j, i}\right)\right) & =\omega_{, i} \\
R\left(1+c \lambda f_{2}\right) w+2 \lambda \frac{\partial}{\partial x_{j}}\left(\frac{1}{f_{2}} \theta_{, j}\right) & =0
\end{aligned}
$$


where $\omega$ is a Lagarange multiplier. Taking the double curl of equation $(3.1)_{1}$ and adopting normal mode representations yields the sixth order governing system

$$
\begin{aligned}
& \frac{2}{\phi}\left(D^{2}-a^{2}\right)^{2} w+2 D^{2}\left(\frac{1}{\phi}\right)\left(D^{2}+a^{2}\right) w+4 D\left(\frac{1}{\phi}\right) D\left(D^{2}-a^{2}\right) w- \\
& 2 D f_{1} D w-2 f_{1}\left(D^{2}-a^{2}\right) w-a^{2} R\left(1+c \lambda f_{2}\right) \theta=0 \\
& \frac{2 \lambda}{f_{2}}\left(D^{2}-a^{2}\right) \theta+2 \lambda D\left(\frac{1}{f_{2}}\right) D \theta+R\left(1+c \lambda f_{2}\right) w=0 .
\end{aligned}
$$

This system can now be used to find the critical nonlinear Rayleigh number $R a_{E}$, such that

$$
R a_{E}=\max _{\lambda} \min _{a^{2}} R^{2}\left(a^{2}, \lambda\right) .
$$

Numerical results for the nonlinear energy approach are presented in $\S 4$.

\section{Numerical results}

The numerical results were derived by utilising the compound matrix method, a technique belonging to the family of shooting methods, as described in Straughan \& Walker (1996) and Straughan (2004). These results were then checked using the Chebyshev-tau technique (cf. Dongarra et al. 1996), which is a spectral technique coupled with the QZ algorithm. The parameters, unless stated otherwise, are fixed at $\phi=0.78, \delta=5 \times 10^{-5}, G_{m}=10, \operatorname{Pr}=6$ and $\epsilon_{T}=0.7$.

Figure 3 shows the linear instability thresholds for a variation of $n$ values, where $n$ is the parameter we choose to decide the shape of the function $F$. From Figure 2 we recall that increasing $n$ improves our approximation of the discontinuous physical parameters in Table 1.

Bimodal behaviour, which is expected for certain parameter ranges in fluid/ porous systems (see e.g., Hirata et al. 2007b, Hirata et al. 2009b, Hill \& Straughan 2009), is captured in Figure 3. In this bimodal behaviour, the fluid mode corresponds to the case where the convective flow is mainly confined in the fluid layer, with the porous mode corresponding to where the convective flow occurs in the entire porous region.

It is clear from Figure 3 that for $\hat{d}=0.2$ the fluid mode is significantly affected by changes in $n$, whereas the affect on the porous mode is negligible. As the porous mode is dominant for $\hat{d}=0.4$, the effect on the neutral curve caused by an increase in $n$ beyond 100 is negligible. In all cases $n$ must be increased until the neutral curve converges, but this does suggest that $n$ must be increased more substantially when studying systems for which the fluid mode may be dominant.

Figure 4 shows the neutral curves for a variation of depth ratio $\hat{d}$ values, where the linear instability and nonlinear stability thresholds for the one layer model are represented by solid lines, and the linear instability thresholds for the two layer model (taken from Hill \& Straughan 2009) are given by dashed lines.

In Figure 4 the linear and nonlinear thresholds for the one domain approach are so close that they are indistinguishable graphically, making the region of potential subcritical instabilities very small, demonstrating the suitability of linear theory to predict the physics of the onset of convection. Thus, by adopting the continuous parameter approach, we can construct nonlinear stability thresholds which show excellent agreement with those of the linear instability theory. 


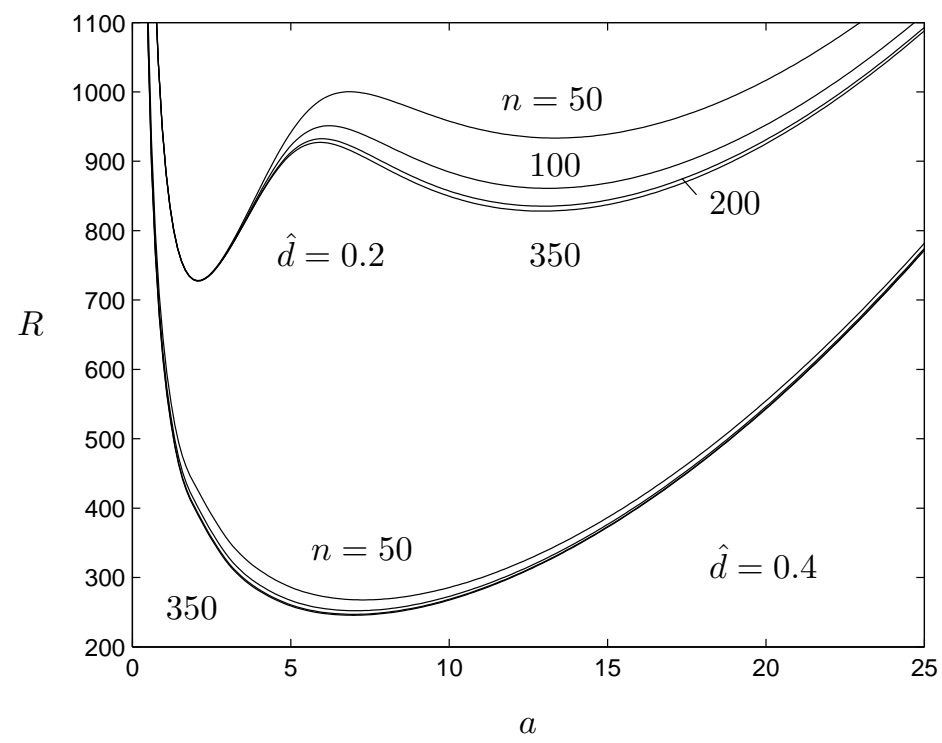

Figure 3. Visual representation of the linear instability thresholds, with critical thermal Rayleigh number $R$ plotted against wavenumber $a$, for $n=50,100,200,350$ and where $\hat{d}$ $=0.2,0.4$.

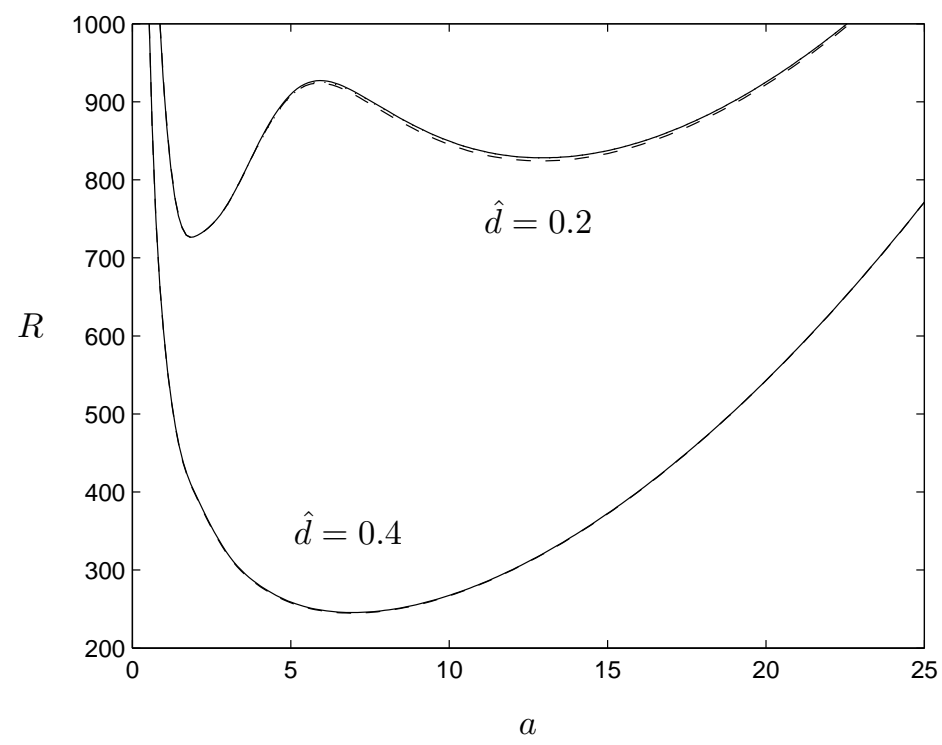

Figure 4. Visual representation of the linear instability and nonlinear stability thresholds of the one layer model (solid lines) and linear thresholds for the two layer model (dashed lines), with critical thermal Rayleigh number $R$ plotted against wavenumber $a$, for $\hat{d}=$ $0.2,0.4$ and where $n=350$. 
Comparing the one layer approach with that of the two domain in Figure 4, we can see that the thresholds show good agreement, demonstrating that the continuous parameter approach captures the behaviour as modelled by the more widely used two domain model.

Since the nonlinear thresholds are essentially the same as the linear ones (for the parameter ranges explored), an exploration of the various physical parameter effects on the stability bound may be found in those published for linear theory, see e.g. Hirata et al. (2007b, 2009a), Hill \& Straughan (2009).

\section{References}

Alazmi, B. \& Vafai, K. 2000 Analysis of variants within the porous media transport models. ASME J. Heat Transfer 122, 303-326.

Alazmi, B. \& Vafai, K. 2001 Analysis of fluid flow and heat transfer interfacial conditions between a porous medium and a fluid layer. Int. J. Heat Mass transfer 44, 1735-1749.

Chandesris, M. \& Jamet, D. 2006 Boundary conditions at a planar fluid-porous interface for a Poiseuille flow. Int. J. Heat Mass Trans. 49, 2137-2150.

Chandesris, M. \& Jamet, D. 2009 Jump Conditions and Surface-Excess Quantities at a Fluid/Porous Interface: A Multi-scale Approach. Trans, Porous Media 78, 419-438.

Chang, M.H. 2006 Thermal convection in superposed fluid and porous layers subjected to a plane Poiseuille flow. Phys Fluids 18, 035104.

Dongarra, J. J., Straughan, B. \& Walker, D.W. 1996 Chebyshev tau-QZ algorithm methods for calculating spectra of hydrodynamic stability problems. App. Num. Math. 22, 399-434.

Goyeau, B., Lhuillier, D., Gobin, D. \& Velarde M. G. 2003 Momentum transport at a fluid-porous interface. Int. J. Heat Mass Trans. 46, 4071-4081.

Hill, A. A. \& Straughan, B. 2009 Global stability for thermal convection in a fluid overlying a highly porous material. Proc Roy Soc A 465, 207-217.

Hill, A.A. \& Carr, M. 2010 Sharp global nonlinear stability for a fluid overlying a highly porous material. Proc R. Soc. A 466, 127-140.

Hirata, S. C., Goyeau, B. \& Gobin, D. 2007a Stability of natural convection in superposed fluid and porous layers: Influence of the interfacial jump boundary condition. Phys Fluids 19, 058102.

Hirata, S. C., Goyeau, B., Gobin, D., Carr, M. \& Cotta, R. M. 2007b Linear stability of natural convection in superposed fluid and porous layers: influence of the interfacial modelling. Int. J. Heat Mass Transfer 50, 1356-1367.

Hirata, S.C., Goyeau, B. \& Gobin, D. 2009a Stability of thermosolutal natural convection in superposed fluid and porous layers. Transp. Porous Med. 78, 525-536.

Hirata, S.C., Goyeau, B., Gobin, D., Chandesris, M. \& Jamet D. 2009b Stability of natural convection in superposed fluid and porous layers: Equivalence of the one- and twodomain approaches. Int. J. Heat Mass Trans. 52, 533-536.

Ladyzhenskaya, O. A. 1969 The mathematical theory of viscous incompressible flow, 2nd edn. New York: Gordon and Breach.

Nield, D. A. \& Bejan, A. 2006 Convection in porous media, 3rd edn. New York: SpringerVerlag.

Ochoa-Tapia, J. A. \& Whitaker, S. 1995 Momentum transfer at the boundary between a porous medium and a homogeneous fluid I. Theoretical development. Int. J. Heat Mass Trans. 38, 26352646.

Payne, L. E. \& Straughan, B. 1998 Analysis of the boundary condition at the interface between a viscous fluid and a porous medium and related modelling questions. J. Math. Pures Appl. 77, 317-354. 
Straughan, B. 2004 The energy method, stability and nonlinear convection. New York: Springer.

Straughan, B. 2008 Stability and wave motion in porous media. Appl. Math. Sci. Ser. vol. 165. New York: Springer.

Straughan, B. \& Walker, D.W. 1996 Two very accurate and efficient methods for computing eigenvalues and eigenfunctions in porous convection problems. J. Computat. Phys. 127, 128-141.

Vafai, K. 2005 Handbook of Porous Media. 2nd edition, New York: Taylor \& Francis. 